\title{
Produção de pneumotórax em cães e manejo por toracoscopia paraxifóide transdiafragmática
}

\author{
Production of pneumothorax in dogs and treatment by transdiaphragmatic paraxiphoid thoracoscopy
}

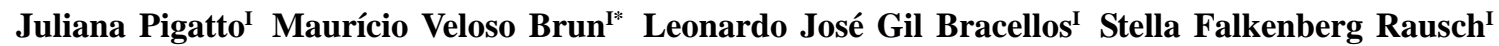 \\ Virgínia Heinze Phol ${ }^{I I}$ João Pedro Scussel Feranti ${ }^{I}$ Rogério Luizari Guedes ${ }^{\text {II }}$
}

\section{RESUMO}

O presente estudo foi desenvolvido com o objetivo de avaliar a técnica de toracoscopia paraxifóide transdiafragmática no diagnóstico e no tratamento do pneumotórax produzido experimentalmente em cães. Para tanto, foram utilizados 11 cães que foram submetidos à produção de pneumotórax grave a partir da aplicação de $10 \mathrm{mLg}^{-1}$ de ar em cada hemitórax até apresentarem descompensação hemodinâmica. Concomitantemente, foram aferidas a correlação entre a pressão venosa central (PVC) e o volume de ar introduzido $\left(\mathrm{mL} \mathrm{kg} \mathrm{k}^{-1}\right)$, bem como FC, FR, TPC, $\mathrm{SpO}_{2}$ e coloração das mucosas. O pneumotórax foi tratado pela aplicação de dreno torácico por meio de um trocarte inserido no lado direito (seis animais) ou esquerdo (cinco animais) do apêndice xifóide por meio do diafragma. A introdução em volume igual ou superior a $50 \mathrm{ml} \mathrm{kg}^{-1}$ hemitórax ${ }^{1}$ de ar causou descompensação cardiorrespiratória e elevação da PVC acima de $10 \mathrm{~cm} \mathrm{H}_{2} \mathrm{O}$ em todos os pacientes. A técnica proposta permitiu apropriado exame da cavidade torácica $e$ aplicação do dreno com efetiva drenagem, sem a ocorrência de complicações trans e pós-operatórias, condição confirmada pela toracoscopia intercostal aos 15 dias de pós-operatório. Conclui-se que o modelo de produção do pneumotórax e a técnica de colocação de dreno proposta para o manejo dessa doença são adequados para cães.

Palavras-chave: toracoscopia, dreno, tórax, canino.

\section{ABSTRACT}

The aim of the present study was to assess the use of transdiaphragmatic paraxiphoid thoracoscopy for the diagnosis and treatment of experimentally induced pneumothorax in dogs. Severe pneumothorax was induced in 11 dogs by the insufflation of $10 \mathrm{~mL} \mathrm{~kg}$ of air into each hemithorax until they became hemodynamically unstable. The correlation between central venous pressure (CVP) and the volume of injected air $\left(\mathrm{mL} \mathrm{kg}^{-1}\right)$ was determined, and was considered too heart rate, respiratory frequency, capillary refill time, oxygen saturation and the color of mucous membranes. Pneumothorax was treated with chest tube drainage with the placement of a trocar into the right side (six dogs) or into the left side (five dogs) of the xiphoid appendix through the diaphragm. The introduction of air volume equal to or greater than $50 \mathrm{ml} \mathrm{kg}^{-1}$ hemitórax ${ }^{-1}$ caused cardiorespiratory decompensation and increased the CVP to levels higher than $10 \mathrm{cmH} O$ in all patients. Transdiaphragmatic paraxiphoid thoracoscopy allowed for adequate examination of the chest cavity and for effective drainage without any intraoperative and postoperative complications, as confirmed by intercostal thoracoscopy 15 days after surgery. The method for induction of pneumothorax and the chest tube placement technique proposed for its management are appropriate to be used in dogs.

Key words: thoracoscopic, drain, torax, canine.

\section{INTRODUÇÃO}

O pneumotórax é definido como o acúmulo de ar no espaço pleural (ANDRADE FILHO et al., 2006), podendo ser traumático, iatrogênico ou espontâneo (ARON \& ROBERTS, 1998). A forma traumática é a mais comum em cães (FOSSUN et al., 2002), sendo que cerca de $47 \%$ dos animais que sofrem trauma torácico apresentam a referida doença (BEAL, 2004). O pneumotórax pode ser classificado como aberto ou fechado (ARON \& ROBERTS, 1998). O primeiro está

ICurso de Medicina Veterinária da Faculdade de Agronomia e Medicina Veterinária (FAMV) da Universidade de Passo Fundo (UPF), Passo Fundo, RS, Brasil. E-mail: mbrum@upf.br.*Autor para correspondência.

IIPrograma de Pós-graduação em Medicina Veterinária da Universidade Federal de Santa Maria (UFSM), Santa Maria, RS, Brasil. 
relacionado a traumas penetrantes no tórax e corresponde ao acúmulo progressivo de ar no espaço pleural proveniente de uma comunicação com o ambiente externo, o qual gera pressão intrapleural positiva e influxo de ar entre as pleuras quando o animal inspira (FOSSUN et al., 2002). Em contrapartida, o fechado geralmente é decorrente de trauma contuso e o acúmulo de ar ocorre devido ao extravasamento a partir do parênquima pulmonar, da árvore brônquica, da traquéia ou do esôfago lesionados (ORTON, 1998).

O pneumotórax fechado é subdividido em simples e de tensão. No simples ocorre acúmulo de ar não-progressivo na cavidade pleural, decorrente de pequenas lacerações no parênquima e cápsula pulmonares (ARON \& ROBERTS, 1998). Já no pneumotórax de tensão, também denominado hipertensivo, observa-se acúmulo progressivo de ar em virtude de lesão extensa no pulmão, a qual cria válvula de direção única, aumentando a pressão intrapleural e levando ao colapso pulmonar. Constitui emergência médica na medida em que a elevação da pressão ocasiona a compressão dos vasos principais, e diminuição do retorno venoso e do débito cardíaco, levando ao desenvolvimento de insuficiência respiratória progressiva, síncope e morte rápida (RAISER, 1999; ANDRADE FILHO et al., 2006).

O pneumotórax espontâneo ocorre na ausência de trauma torácico, sendo classificado em primário e secundário. Primariamente ocorre em pacientes sem doença pulmonar evidente, geralmente, decorrente do rompimento de bolhas subpleurais pela alta pressão negativa intratorácica (BAUMANN et al., 2001). Complicações decorrentes de pneumonias, abscessos pulmonares, neoplasias, infecções granulomatosas crônicas e parasitas pulmonares desencadeiam pneumotórax espontâneo secundário (FOSSUN et al., 2002).

Segundo AGUIAR et al. (2004), a pressão venosa central (PVC) é a pressão de retorno do sangue ao lado direito do coração e é importante parâmetro a ser aferido em numerosas situações clínicas, cirúrgicas e experimentais. Os valores normais da PVC em cães e gatos podem situar-se entre -2 e $10 \mathrm{~cm} \mathrm{H}_{2} \mathrm{O}$ (HAUPTMAN \& CHAUDRY,1998; RAISER, 2005; WALTON, 1998). No caso de pneumotórax, efusão pleural e hérnia diafragmática, os valores da PVC encontram-se elevados em função da pressão intrapleural aumentada, a qual comprime as veias cavas, impedindo o retorno venoso adequado (RAISER, 2005).

O tratamento clínico de pacientes com pneumotórax consiste, inicialmente, no alívio da dispnéia por meio de toracocentese com agulha. $\mathrm{O}$ colapso pulmonar superior a $25 \%$ é considerado um pneumotórax de moderado a grave e, freqüentemente, é tratado com dreno de tórax (ARON \& ROBERTS, 1998). CROWE \& DEVEY (2002), indicam a colocação do dreno quando ocorrer produção maior que $10 \mathrm{~mL} . \mathrm{kg}^{-}$ ${ }^{1}$ de ar no espaço pleural num período de 12 horas. Já o tratamento do pneumotórax espontâneo e progressivo exige, muitas vezes, ampla exploração da cavidade torácica na busca da causa e possível correção do defeito.

As técnicas tradicionais de exploração da cavidade torácica incluem as toracotomias, que são técnicas muito invasivas, o que aumenta a morbidade e a mortalidade dos pacientes no trans e pósoperatório, e as toracoscopias, que são acessos de invasão mínima, os quais reduzem o trauma da abordagem convencional sem reduzir a exposição cirúrgica ou diminuir a qualidade do procedimento, permitindo rápida recuperação do paciente e reduzida dor pós-operatória (POTTER \& HENDRICKSON, 1998).

A toracoscopia é indicada para exploração geral da cavidade torácica e para procedimentos como biópsias pulmonares, lobectomia, pericardiectomia e ligadura da persistência do canal arterial (TWEDT, 2002). VACHON \& FISCHER (1998) citaram que a toracoscopia é recomendada para colocação de drenos por contribuir para a melhor orientação espacial em razão da localização de massas e abscessos, aumentando a segurança do procedimento. Entre os acessos descritos para toracoscopia encontram-se o cervical, o intercostal e o paraxifóide transdiafragmático (POTTER \& HENDRICKSON, 1998).

O acesso paraxifóide transdiafragmático é descrito para procedimentos de pericardiectomia parcial em pacientes que apresentam efusão pericárdica. Leva vantagem sobre os demais, pois permite ampla exploração da cavidade torácica em ambos os hemitórax, bem como ótima visibilização do hilo pulmonar, do mediastino e das estruturas ventrais do tórax. Os demais acessos permitem somente a exploração do hemitórax correspondente, podendo acarretar atraso na identificação de lesões que estão localizadas no hemitórax contra lateral (POTTER \& HENDRICKSON, 1998).

Nesse contexto, o presente estudo objetivou avaliar o acesso toracoscópico na terapêutica cirúrgica do pneumotórax grave produzido experimentalmente em cães, a partir da aplicação do dreno de tórax transdiafragmático, bem como correlacionar o volume máximo de ar em $\mathrm{ml} \mathrm{kg}^{-1}$ infundidos em cada hemitórax capaz de elevar a PVC acima de $10 \mathrm{cmH}_{2} \mathrm{O}$ e causar alterações hemodinâmicas descompensatórias. 


\section{MATERIAL E MÉTODOS}

No presente estudo foram utilizados 11 cães, fêmeas, sem raça definida, com massa corporal média de 10,1 $\pm 0,95 \mathrm{~kg}$, provenientes do Biotério da Universidade de Passo Fundo. Todas as etapas do trabalho foram realizadas seguindo os princípios éticos na experimentação animal preconizados pelo COBEA, tendo a aprovação pelo comitê de ética da UPF (protocolo 047/2008), sendo todos os caninos submetidos à ovariosalpingohisterectomia e doados ao final do experimento.

Todos os animais foram avaliados clinicamente, desverminados e alojados em canis individuais, onde receberam ração comercial e água $\mathrm{ad}$ libitum. O experimento foi dividido em três etapas, a citar: a) indução do pneumotórax, b) tratamento do pneumotórax e c) avaliação pós-operatória. Na primeira etapa, os animais foram pré-medicados com maleato de acetilpromazina $\left(0,05 \mathrm{mg} \mathrm{kg}^{-1}\right.$, IM) e sulfato de morfina $\left(0,5 \mathrm{mg} \mathrm{kg}^{-1}, \mathrm{IM}\right)$. Após 15 minutos, a anestesia geral foi induzida com diazepan $\left(0,5 \mathrm{mg} \mathrm{kg}^{-1}\right.$, IV) e tiopental sódico $\left(5 \mathrm{mg} \mathrm{kg}^{-1}, \mathrm{IV}\right)$. A manutenção anestésica foi obtida com isofluorano vaporizado em oxigênio a $100 \%$, em circuito semifechado, com respiração assistida.

Posteriormente, foi posicionado um cateter venoso central (19G) na veia jugular direita de cada animal, o qual foi heparinizado e fixado à pele com pontos isolados simples com fio de náilon 3-0. Os equipos para mensuração da Pressão Venosa Central (PVC) foram montados em haste de suporte para fluidos e uma régua com escala em $\mathrm{cm}$ foi posicionada com o seu ponto zero ao nível do átrio direito. Para tanto, os animais foram posicionados em decúbito dorsal e o manúbrio foi palpado como ponto de referência, sendo utilizada uma régua nivelada para evitar desvios. Foi utilizada uma dânula para conexão envolvendo o frasco de fluido, a coluna de água e o paciente, sendo administrado Ringer com Lactato de Sódio na velocidade de $10 \mathrm{~mL} \mathrm{~kg}^{-1} \mathrm{~h}^{-1}$.

Os animais foram submetidos a uma toracocentese com escalpe $\left(n^{\circ} 21\right)$ no sétimo espaço intercostal bilateralmente e, com o auxílio de uma seringa de $60 \mathrm{~mL}$ e uma dânula, foram introduzidos $10 \mathrm{~mL}$ $\mathrm{kg}^{-1} \mathrm{de}$ ar por vez em cada hemitórax, com o objetivo de induzir pneumotórax e avaliar os parâmetros da PVC, a frequiência cardíaca (FC), a freqüência respiratória (FR), o padrão respiratório (PR), a coloração das mucosas, o tempo de perfusão capilar (TPC) e a saturação de oxigênio a cada aplicação $\left(\mathrm{SpO}_{2}\right)$. O pneumotórax foi induzido até os cães iniciarem com respiração agônica, sendo então drenados $10 \mathrm{~mL} \mathrm{~kg}^{-1}$ de ar de cada hemitórax, procedendo-se ventilação pulmonar até a estabilização dos mesmos. Na seqüência, realizou-se radiografia torácica para confirmar e avaliar o grau de pneumotórax induzido. As medidas da PVC foram tomadas no momento da indução do pneumotórax, a cada aplicação de ar, considerando-se: Volume um (V1) $=10 \mathrm{~mL} \mathrm{~kg}^{-1}, \mathrm{~V} 2=20 \mathrm{~mL} \mathrm{~kg}^{-1}$, assim sucessivamente até chegar ao valor descompensatório. Tal parâmetro também foi avaliado em cinco momentos cirúrgicos: antes da entrada do trocarte (M1), após a entrada do trocarte (M2), após a colocação do dreno (M3), após a drenagem da cavidade (M4) e após a retirada do dreno (M5).

Na segunda etapa do experimento, o animal foi posicionado em decúbito dorsal, realizando-se incisão com bisturi de aproximadamente $0,5 \mathrm{~cm}$ na pele entre o apêndice xifóide e o arco costal, que serviu para a introdução do trocarte de $5 \mathrm{~mm} \varnothing$. A cânula, após trajeto pelo subcutâneo de aproximadamente $2 \mathrm{~cm}$, foi direcionada craniolateralmente através do diafragma no hemitórax selecionado (em cinco cães no esquerdo e seis no direito). $O$ endoscópio rígido de $4 \mathrm{~mm} \varnothing$ e $25^{\circ}$ foi introduzido através do trocarte, dando-se início à exploração do hemitórax correspondente. Posteriormente, quando necessário, perfurou-se a membrana mediastínica com o próprio instrumento, procedendo-se à exploração no hemitórax contralateral. Na seqüência, um dreno de tórax (10FR) foi posicionado através do trocarte de $5 \mathrm{~mm} \varnothing$, sem a válvula, no hemitórax correspondente à incisão. $\mathrm{O}$ trocarte foi removido, o local de punção na pele obliterado e o dreno fixado com fio de seda 2-0 em padrão de sutura chinesa. Ao final do procedimento, a pressão torácica negativa foi restabelecida através de uma dânula e seringa de $60 \mathrm{~mL}$ acopladas ao dreno de tórax. Foi realizado outro estudo radiográfico para avaliar a resolução do pneumotórax e o posicionamento do dreno. O dreno foi então retirado em movimento único, sendo o local ocluido com a sutura de fixação previamente aplicada. No pós-operatório foi administrado cloridrato de tramadol ( $2 \mathrm{mLkg}^{-1}$, IM, BID) e cetoprofeno ( $2 \mathrm{mg} \mathrm{kg}^{-1}$, IM, SID), ambos por três dias, sendo que a higiene da ferida cirúrgica foi feita com $\mathrm{NaCl}$ a $0,9 \%$, até a retirada das suturas aos sete dias de evolução.

A terceira etapa do experimento foi realizada 15 dias após o procedimento cirúrgico, com o objetivo inicial de explorar a cavidade torácica na busca de alterações relacionadas com a técnica e avaliar a condição do defeito diafragmático através de toracoscopia intercostal no lado correlacionado à introdução do dreno. Após a avaliação pré-operatória e indução anestésica, os animais foram posicionados em decúbito dorsal e o hemitórax correspondente ao 
defeito diafragmático foi preparado para cirurgia asséptica. Uma incisão de aproximadamente $0,5 \mathrm{~cm}$ foi feita no 6 o espaço intercostal na altura da junção costocondral, abrangendo a pele, o tecido subcutâneo, a musculatura e a pleura parietal. Pela abertura, o trocarte de $5 \mathrm{~mm} \varnothing$ foi introduzido e o endoscópio rígido foi acoplado dando início à inspeção da cavidade em busca de possíveis complicações relacionadas com o procedimento anterior. Promoveu-se então avaliação criteriosa do defeito diafragmático. Ao final da inspeção, o trocarte foi removido, a incisão obliterada com fio de náilon 3-0, em padrão de sutura isolado simples, e a pressão torácica negativa restabelecida com escalpe.

As variáveis de medida analisadas neste estudo foram a correlação dos valores da PVC com o volume de ar introduzido ( $\mathrm{mL} \mathrm{kg}^{-1}$ ), as alterações nos parâmetros fisiológicos da FC, FR, PR, TPC, $\mathrm{SpO}_{2}$ e a coloração das mucosas, conforme o volume de ar introduzido, sendo os dados analisados por teste Anova, seguido de Tukey, considerando as diferenças como significativas quando $\mathrm{P}<0,05$. A funcionabilidade de aplicação do dreno de tórax foi avaliada pela redução nos valores da PVC e pelo estudo radiográfico, sendo a eficácia da toracoscopia avaliada pelo tempo cirúrgico, pela ausência de complicações, pela drenagem eficiente e pelo selamento do defeito diafragmático.

\section{RESULTADOS E DISCUSSÃO}

O protocolo anestésico utilizado no experimento foi adequado, pois foi constatada mínima depressão cardiorespiratória em 10 dos 11 animais até o momento da descompensação pelo pneumotórax e todas as toracoscopias ocorreram sem incidentes. Destacou-se um caso de parada cardiorespiratória reversível após introdução de $50 \mathrm{~mL} \mathrm{~kg}^{-1}$ de ar em cada hemitórax, condição que será posteriormente discutida.

O modelo proposto para obtenção do pneumotórax foi efetivo em todos os animais, condição confirmada pelas radiografias que demonstraram a elevação do coração em relação ao esterno, aumento da opacidade dos lobos pulmonares atelectásicos e a hiperluscência do campo pulmonar de ambos hemitórax (Figura 1A), achados previamente observados por ARON \& ROBERTS (1998) eNELSON \& COUTO(2001). Dos 11 animais utilizados para a avaliação da técnica proposta, somente em oito foram considerados os valores da PVC, visto que nos outros houve interferência na sua leitura. Em um dos animais houve deslocamento do cateter para dentro do espaço pleural, levando ao hidrotórax. Esta é uma das complicações observadas em humanos, com o uso do CVC, sendo mais comum em crianças (PELICANO et al., 2005). O mesmo autor também cita a punção da artéria carótida, o pneumotórax, o hemotórax, o tamponamento cardíaco, a bacteremia, a embolia e a sepse como outras complicações relacionadas com o uso do cateter por períodos prolongados, condições não observadas no presente estudo, já que o tempo de permanência do cateter foi curto. Salienta-se a importância da toracoscopia no diagnóstico do mau posicionamento do cateter no animal desse experimento, pois as mensurações em relação a sua suposta PVC aumentavam gradativamente durante a progressão do pneumotórax, constatando um resultado falso positivo de elevação desse parâmetro. Nesse caso havia sido aferida, na verdade, a pressão intratorácica secundária ao hidro e pneumotórax instaurado.

Os valores da PVC aferidos antes do início da indução do pneumotórax em todos os animais $\left(0,45 \pm 1,8 \mathrm{cmH}_{2} \mathrm{O}\right)$ encontraram-se dentro do padrão considerado normal por RAISER (2005), que varia de -2 a $4 \mathrm{cmH}_{2} \mathrm{O}$, o que demonstra que os pacientes estavam em situação hemodinâmica estável. As médias da PVC obtidas a cada aplicação de $10 \mathrm{~mL} \mathrm{~kg}^{-1}$ de ar, considerado V0 $\left(0 \mathrm{~mL} \mathrm{~kg}{ }^{-1}\right)$ até V7 $\left(70 \mathrm{~mL} \mathrm{~kg}^{-1}\right)$, demonstraram um aumento discreto de $\mathrm{V} 0=0,45 \pm 1,8 \mathrm{cmH}_{2} \mathrm{O}$ até $\mathrm{V} 4=3,76 \pm 2,48 \mathrm{cmH}_{2} \mathrm{O}$. Em seguida, observou-se um aumento estatisticamente significativo a partir de $\mathrm{V} 5=6,46 \pm 4,42 \mathrm{cmH}_{2} \mathrm{O}$ até $\mathrm{V7}=12,96 \pm 2,16 \mathrm{cmH}_{2} \mathrm{O}$ em relação à primeira avaliação da PVC. As mensurações obtidas demonstraram elevação gradativa da PVC em função do aumento gradativo da pressão intrapleural, secundária ao pneumotórax instaurado, o qual dificultou o retorno venoso pelo efeito de ocupação de espaço (RAISER, 2005). Isso refletiu na variação da pressão intratorácica, que é um dos determinantes básicos da PVC (WALTON, 1998). A associação da PVC com os sinais de descompensação, neste experimento, ocorreu a partir da aplicação de $50 \mathrm{~mL} \mathrm{~kg}^{-1}$ de ar ou mais, atingindo valores iguais ou superiores a $10 \mathrm{cmH}_{2} \mathrm{O}$. No presente trabalho, considerou-se PVC dessa magnitude como sendo de alto risco para os cães, discordando de demais relatos que afirmam que o valor de $10 \mathrm{~cm} \mathrm{H}_{2} \mathrm{O}$ é considerado normal (HENDRIX \& RAFFE, 1998; WALTON, 1998). Ainda em relação a PVC, entre os oito animais, dois (25\%) descompensaram após introdução de $50 \mathrm{~mL} \mathrm{~kg}^{-1}$ de ar, um (12\%) aos $60 \mathrm{~mL} \mathrm{~kg}^{-1} \mathrm{e}$ cinco $(63 \%)$ aos $70 \mathrm{~mL} \mathrm{~kg}^{-1}$. Todos pacientes, ao descompensarem, iniciaram respiração agônica, apresentando valores da PVC iguais ou superiores a $10 \mathrm{~cm} \mathrm{H}_{2} \mathrm{O}$, destacando-se que todos aqueles que suportaram $70 \mathrm{~mL} \mathrm{~kg}^{-1}$ de ar em cada hemitórax possuíam tórax profundo. Durante o procedimento cirúrgico de resolução do pneumotórax, notou-se queda significativa na PVC em M1=9,35 $\pm 2,93 \mathrm{~cm} \mathrm{H}_{2} \mathrm{O}$ 


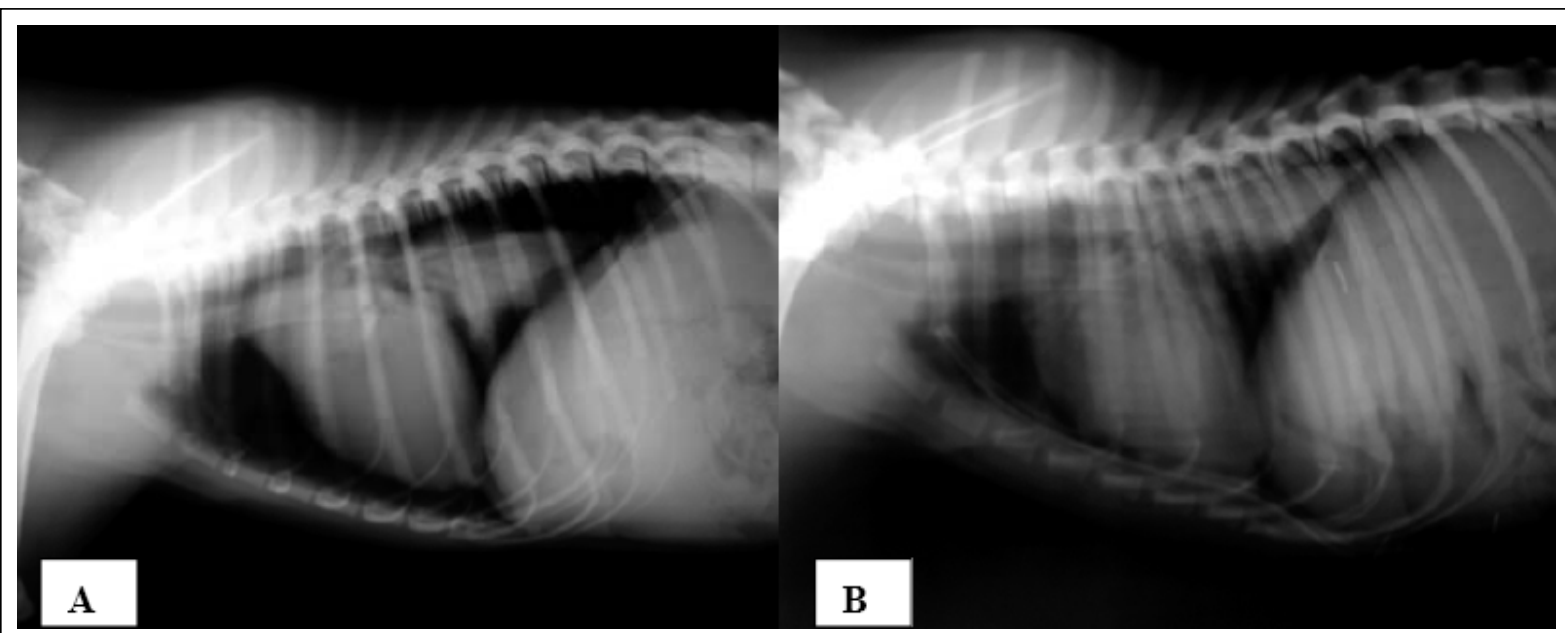

Figura 1 - A) Imagem demonstrando pneumotórax grave, com elevação do coração em relação ao esterno, aumento da opacidade do pulmão atelectásico e hiperluscência do campo pulmonar. B) Imagem demonstrando dreno de tórax na posição ventral, apoiado sob o esterno após efetiva drenagem da cavidade pela toracoscopia paraxifóide transdiafragmática.

até $\mathrm{M} 4=0,48 \pm 2,75 \mathrm{~cm} \mathrm{H}_{2} \mathrm{O}$, estabilizando em $\mathrm{M} 5=0,11 \pm 1,55 \mathrm{~cm} \mathrm{H}_{2} \mathrm{O}$. Isso confirma o mecanismo de elevação da PVC citado anteriormente, uma vez que, com a remoção do ar, a redução desse parâmetro foi evidente. Por ocasião da drenagem até a retirada do dreno, os valores desse parâmetro estiveram dentro da normalidade, o que demonstra a efetividade do tratamento cirúrgico proposto.

Durante a indução do pneumotórax observou-se, nos 11 animais avaliados, que houve acréscimo nos valores da FC e FR, atingindo o ponto máximo com a introdução de $50 \mathrm{~mL} \mathrm{~kg}^{-1}$ de ar. A partir daí, houve um decréscimo em nove cães, os quais suportaram $70 \mathrm{~mL} \mathrm{~kg}^{-1}$ por hemitórax de ar. A taquicardia e a taquipnéia presentes durante a progressão do pneumotórax demonstram o mecanismo compensatório do choque instaurado nos animais, sendo que o obstáculo circulatório acabou por restringir o retorno venoso à grande circulação, levando à diminuição do débito cardíaco (RAISER, 2005). Quando a magnitude e a duração do insulto ultrapassaram a capacidade de regulação homeostática, instalou-se quadro de insuficiência circulatória e respiratória aguda (WALTON, 1998), levando à queda acentuada e contínua dos batimentos cardíacos e dos movimentos respiratórios, e tornando necessária a estabilização destes por ventilação assistida, a fim de evitar a parada cardiorespiratória. Ainda assim, essa condição ocorreu em um animal após introdução de $70 \mathrm{~mL} \mathrm{~kg}^{-1}$ de ar, sendo responsiva à massagem cardíaca externa e à aplicação de $0,1 \mathrm{~mL} \mathrm{~kg}^{-1} \mathrm{de}$ adrenalina intratraqueal na concentração de 1:10000.

As demais alterações nos parâmetros fisiológicos em relação ao pneumotórax foram: aumento no tempo de perfusão capilar de 1 segundo para 3 segundos em 11 (100\%) dos animais após a infusão de $30 \mathrm{~mL} \mathrm{~kg}^{-1}$ de ar; a alteração no padrão respiratório, o qual passou a ser restritivo a partir da infusão de $30 \mathrm{~mL}$ $\mathrm{kg}^{-1}$ em dois (18\%) dos cães, de 40 $\mathrm{mL} \mathrm{kg}^{-1}$ em três (27\%) e de $50 \mathrm{~mL} \mathrm{~kg}^{-1}$ em seis (55\%) dos animais; a coloração das membranas mucosas passou a ser cianótica em 100\% dos pacientes no momento da descompensação, juntamente com o início da respiração agônica. Dos 11 animais, dois (18\%) desenvolveram sinais evidentes de choque obstrutivo após introdução de $50 \mathrm{~mL} \mathrm{~kg}^{-1} \mathrm{de}$ ar, dois (18\%) aos 60 $\mathrm{mL} \mathrm{kg}^{-1}$ e sete (64\%) aos $70 \mathrm{~mL} \mathrm{~kg}^{-1}$. A saturação de oxigênio foi caindo lentamente com a progressão do pneumotórax e caiu bruscamente com o início da agonia respiratória. Diferentemente do observado nesse estudo, acredita-se que, em condições não-controladas de fornecimento de oxigênio, os animais com pneumotórax descompensariam antes de atingir volumes máximos suportados, de tal forma que tais quantidades de ar por hemitórax não devem ser consideradas como referência para casos clínicos, ainda mais que poderão coexistir outras alterações hemodinâmicas.

$\mathrm{O}$ procedimento relacionado à segunda etapa do experimento resultou em tempo cirúrgico

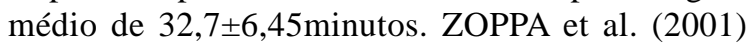
acreditam que o tempo necessário para inspeção adequada da cavidade torácica de eqüinos pode ser inferior a 30 minutos. O acesso paraxifóide transdiafragmático, com os animais em decúbito dorsal, mostrou-se efetivo, porém, os autores deste trabalho ressaltam o cuidado para que a angulação de entrada do trocarte em relação ao esterno seja inferior a $45^{\circ}$, condição a qual se atribui menor chance de lesão ao 
coração e pulmão e facilidade quanto à colocação correta do dreno de tórax. Não houve diferenças quanto à entrada do trocarte pelo hemitórax direito ou esquerdo; contudo, considera-se que há uma maior possibilidade de lesionar o ápice do coração com a introdução pelo lado esquerdo, já que esse órgão é direcionado em aproximadamente $30^{\circ}$ para a esquerda de linha média.

Após inserção do trocarte, foi necessário romper a pleura mediastínica na maioria dos casos, para posterior exploração do hemitórax contralateral, pois esta se apresentava íntegra em $82 \%$ dos animais. Considera-se que cães e gatos possam apresentar comunicação natural entre os hemitórax (ORTON, 1998), o que também foi observado no presente trabalho. Indica-se, então, para casos de pneumotórax unilateral, a entrada do trocarte pelo lado afetado, mesmo sendo possível avaliar ambos os lados pela ruptura do mediastino, já que o pulmão correspondente estará colabado, o que possibilita maior segurança quanto a lesões iatrogênicas. Caso a doença seja bilateral, recomenda-se a entrada no hemitórax direito, devido à angulação do coração.

O acesso toracoscópico proposto para a colocação do dreno permitiu a ampla exploração da cavidade torácica, conforme relatam POTTER \& HENDRICKSON (1998), bem como ótima visibilização do parênquima e hilo pulmonar, mediastino e outras estruturas torácicas como: aorta; artéria subclávia esquerda e direita; veia costocervical direita; veia ázigos direita; veia cava cranial e caudal; veias, nervos e músculos intercostais; costelas e diafragma. O nervo frênico foi observado em quatro animais, sendo que o esôfago e o nervo vago não foram observados. Esses achados estão de acordo com o relatado de TWEDT (2002), com exceção do nervo vago e do esôfago, que, conforme o autor citado, seriam de fácil observação. Em um animal foram observados quatro calos ósseos junto às costelas localizadas no hemitórax direito e presença de filetes de secreção mucóide, possivelmente associados à pleurite e à aderência do lobo pulmonar acessório no médio. Isso advoga quanto à importância da toracoscopia como ferramenta diagnóstica (POTTER \& HENDRICKSON, 1998; ZOPPAetal., 2001; TWEDT, 2002).

Segundo as observações do presente estudo, a abordagem paraxifóide transdiafragmática pode ser considerada viável para procedimentos selecionados de emergência, bem como em casos de trauma torácico associado a fraturas de costelas e pneumotórax progressivo, visto que nessa doença torna-se necessária a observação de lesões no parênquima de todos os lobos pulmonares, estando contra-indicada a colocação de dreno pela musculatura intercostal, pois poderia gerar o deslocamento medial das esquírolas costais, incorrendo lesão ao pulmão ou coração (AGUIAR, 2007), além de ocasionar menor invasão que as toracotomias (WALLER, 1997). Apesar de estudos demonstrarem que os índices de complicação da toracotomia e da toracoscopia serem superponíveis (de 8 a 10\%, respectivamente), considerase que, para o fim proposto neste trabalho, a toracotomia, para a inspeção do tórax, por menor que fosse, acarretaria agressão cirúrgica consideravelmente maior. Os autores deste trabalho concordam com WALLER (1997) quanto a sua recomendação do acesso convencional apenas para situações nas quais não existe a disponibilidade dos recursos tecnológicos necessários para a realização de toracoscopia ou então para casos de insucesso com essa técnica.

Logo após a inserção do trocarte, procurouse mensurar visualmente a porcentagem de colabamento dos lobos pulmonares, destacando que $11(100 \%)$ dos animais apresentaram mais de 50\% de atelectasia total, mesmo após a insuflação pulmonar que foi procedida por ocasião da descompensação na primeira etapa do experimento. Durante a toracoscopia, seis (54\%) cães apresentaram mais de $70 \%$ de colabamento de ambos os pulmões, necessitando de ventilação durante toda a toracoscopia para estabilização da saturação de oxigênio. Os achados estão de acordo com os observados por ARON \& ROBERTS (1998), que consideram o colapso pulmonar superior a $25 \%$ um pneumotórax de moderado a grave, sendo indicado o tratamento com dreno de tórax.

A utilização terapêutica do acesso toracoscópico permitiu, com sucesso, a colocação do dreno de tórax na posição ventral, apoiado sob o esterno, em nove (82\%) animais (Figura 1B), posicionamento indicado por ORTON (1998) e NELSON \& COUTO (2001). Contudo, dois (18\%) cães tiveram o dreno posicionado no terço dorsal do tórax, possivelmente pela angulação de entrada do trocarte no tórax ter sido maior que $45^{\circ}$, o que não acarretou prejuízos quanto à drenagem. Isso ratifica quanto à adequabilidade do posicionamento dorsal na remoção de ar, conforme citam ARON \& ROBERTS (1998). Se o objetivo fosse a drenagem de líquidos, essa posição poderia até mesmo ser ineficiente.

Em comparação com a técnica tradicional de colocação do dreno pela musculatura intercostal citada por FOSSUN et al. (2002), considera-se que a técnica proposta seja menos traumática, em virtude da inexistência de agressão à musculatura da parede torácica e à inexistência de fáscia endotorácica, podendo associar a operação com menores perdas de sangue, formação de fibrose, incidência de infecções e incidência de dor pós-operatória. 
A radiografia foi útil na determinação da presença e da gravidade do pneumotórax, para a avaliação do posicionamento do tubo torácico e para o controle do tratamento e a progressão da doença, conforme relatam ARON \& ROBERTS (1998). Todos os animais apresentaram expansão pulmonar e remoção eficiente do ar ao final da drenagem, o que foi confirmado pelo estudo radiográfico do tórax no pósoperatório.

Na terceira etapa, a utilização do método aberto para a introdução do trocarte durante a toracoscopia intercostal foi considerada satisfatória, devido à ausência de lesões iatrogênicas às estruturas presentes no hemitórax selecionado, fato também observado por BECK et al. (2004) em reparos de hérnias diafragmáticas por meio da toracoscopia intercostal. Ao término da exploração do hemitórax, verificou-se presença de aderências da membrana mediastínica na porção muscular e tendínea do diafragma, em 100\% dos animais, não acarretando prejuízos relacionados à ventilação. Não foram encontradas aderências nos lobos pulmonares, nas herniações de órgãos, nos sangramentos ou na fibrose na cavidade, o que reforça a possibilidade de introdução do dreno transdiafragmático, sem ocorrências de lesões intratorácicas, conforme demonstrado por técnica convencional através do flanco em cadáveres (AGUIAR, 2007).

O defeito de $5 \mathrm{~mm}$ produzido pelo trocarte na porção muscular do diafragma, junto a sua inserção no apêndice xifóide, estava selado aos 15 dias do procedimento em todos os animais, nem sempre sendo observado processo cicatricial que, quando localizado, apresentava-se como uma pequena cicatriz. Segundo RAISER (2005), devido ao fato que o diafragma é considerado um músculo esquelético, a cicatriz é secundária à reparação da musculatura, a qual se contrai e forma extensa fibrose nos casos de secção com afastamento dos segmentos. Como no presente estudo o afastamento dos segmentos foi considerado mínimo, a fibrose associada foi de difícil visibilização.

\section{CONCLUSÕES}

O modelo de produção do pneumotórax grave é adequado para cães, sendo que, em animais hígidos, o volume de ar igual ou superior a $50 \mathrm{~mL} \mathrm{~kg}^{-1}$ por hemitórax causa descompensação cardiorespiratória e elevação da PVC acima de $10 \mathrm{~cm} \mathrm{H}_{2} \mathrm{O}$.

A toracoscopia paraxifóide transdiafragmática é adequada para a avaliação da cavidade torácica em pacientes com pneumotórax progressivo e para o tratamento dessa doença por meio da aplicação do dreno pelo mesmo portal de acesso, sendo um procedimento seguro, eficaz e com pouco risco de complicações. Contudo, na presença de pneumotórax bilateral, indica-se o acesso paraxifóide direito para aplicação do dreno, devido à angulação do ápice do coração para a esquerda da linha média.

\section{COMITÊ DE ÉTICA E BIOSSEGURANÇA}

Trabalho aprovado pelo Comitê de Ética e Biossegurança da Universidade de Passo Fundo, sob o protocolo de $n^{\circ} 047 / 2008$.

\section{REFERÊNCIAS}

AGUIAR, E.S.V. et al. Teste de introdução transdiafragmática de drenos torácicos em cadáveres de cães. Ciência Rural, v.37, n.6, p.1708-1711, 2007.

AGUIAR, E.S.V. et al. Mensuração da pressão venosa central por meio de cateteres venosa central e periférico: comparação entre os valores obtidos em cães e elaboração de índice de correção. Ciência Rural, v.34, n.6, p.1827-1831, 2004.

ANDRADE FILHO, L.O. et al. Pneumotórax. J Bras Pneumol, v.32, supl.4, p.212-216, 2006.

ARON, D.N.; ROBERTS, R.E. Pneumotórax. In: BOJRAB, M.J. Mecanismos da moléstia na cirurgia dos pequenos animais. São Paulo: Manole, 1998. Cap.68, p.468-475.

BAUMANN, M.H. et al. Management of spontaneous pneumothorax. An American College of Chest Physicians Delphi Consensus Statement. Chest, v.119, n.2, p.590-602, 2001.

BEAL, M.W. Thoracic trauma: what to anticipate and how to correct it (VET-131). In: WESTERRN VETERINARY CONFERENCE, 2004. Front Page: Library: wvc 2004. Critical care: thoracic trauma. Acessado em 20/07/2007. On line. Disponível em http:/www.vingram@vin.com.

BECK, C.A.C. et al. Toracoscopia nas hérnias diafragmáticas: estudo experimental em cães. Ciência Rural, v.34, n.6, p.1857-1863, 2004.

CROWE, D.T.; DEVEY, J. Pneumothorax: those not so bad - those real bad. In: WESTERN VETERINARY CONFERENCE, 2002. Front page: Library: wVc 2002: Critical care: pneumothorax. Acessado em 15/08/2007. On line. Disponível em: http:// www.vingram@vin.com.

FOSSUN, T.W. et al. Cirurgia do sistema respiratório inferior: cavidade pleural e diafragma. In:______. Cirurgia de pequenos animais. São Paulo: Roca, 2002. Cap.27, p.752785 .

HAUPTMAN, J.; CHAUDRY, I.H. Choque: fisioterapia e tratamento da hipovolemia e infecção/septicemia. In: SLATTER, D. Manual de cirurgia de pequenos animais. São Paulo: Manole, 1998. Cap.1, p.1-12.

HENDRIX, P.K.; RAFFE, M.R. Distúrbios dos líquidos, eletrólitos e ácidobásicos. In: BOJRAB, M.J. Mecanismos da 
moléstia na cirurgia dos pequenos animais. 2.ed. São Paulo: Manole, 1998. Cap.5, p.26-38.

NELSON, R.W; COUTO, C.G. Manifestações clínicas da doença mediastinal e da cavidade pleural. In: Medicina interna de pequenos animais. 2.ed. Rio de Janeiro: Guanabara, 2001. Cap.23, p.247-251.

ORTON, E.C. Pleura e espaço pleural. In: SLATTER, D. Manual de cirurgia de pequenos animais. São Paulo: Manole, 1998. V.1, Cap.32, p.469-489.

PELICANO, J.F. et al. Complicação de acesso venoso central: hidrotórax. Med J, v.123, p.25, 2005.

POTTER, L.; HENDRICKSON, D.A. Therapeutic video-assisted thoracic surgery. In: FREEMAN, L.J. Veterinary endosurgery. Saint Louis: Mosby, 1998. Cap.9, p.169-187.

RAISER, A.G. Choque. In: RABELO, R.C.; CROWE, D.T. Fundamentos de terapia intensive veterinária em pequenos animais. Rio de Janeiro: L.F. Livros de Veterinária, 2005. p.71-104.
RAISER, A.G. Pneumotórx traumático em cães e gatos. Arquivo Brasileiro Veterinária Zootecnia, v.51, n.1, p.5766, 1999.

TWEDT, D.C. Diagnostic toracoscopy. In: WESTERN VETERINARY CONFERENCE 2002. Front page: Library: wvc 2002: endoscopy. Dianostic thoracoscopy. Acessado em 04/01/2007. On line. Disponível em: http:// www.vingram@vin.com.

VACHON, A.M.; FISCHER, A. Thoracoscopy in the horse: diagnostic and therapeutic indications in 28 cases. Equine Veterinary Journal, v.30, n.6, p.467-475, 1998.

WALLER, D.A. Video-assisted thoracoccopic surgery (VATS) in the management of spontaneous pneumothorax. Thorax, v.52, n.4, p.307-308, 1997

WALTON, R.S. Choque. In: WINGFIELD, W.E. Segredos em medicina veterinária. Porto Alegre: Artes Médicas Sul, 1998. Cap.5, p.49-54.

ZOPPA, A.L.V. et al. Toracoscopia em equínos: técnica e emprego como método de avaliação da cavidade pleural. Ciência Rural, v.34, n.5, p.825-830, 2001. 